mitochondrial genome in human colorectal tumours. Nat. Genet. 20:291-293.

4. Taylor, R.W., et al. 2003. Mitochondrial DNA mutations in human colonic crypt stem cells. J. Clin. Invest. 112:1351-1360. doi:10.1172/JCI200319435

5. Jenuth, J.P., Peterson, A.C., Fu, K., and Shoubridge, E.A. 1996. Random genetic drift in the female germline explains the rapid segregation of mammalian mitochondrial DNA. Nat.
Genet 14:146-151.

6. Bonilla, E., et al. 1992. New morphological approaches to the study of mitochondrial encephalomyopathies. Brain Pathol. 2:113-119.

7. Bonilla, E., et al. 1999. Mitochondrial involvement in Alzheimer's disease. Biochim. Biophys. Acta. 1410:171-182.

8. DiMauro, S., Tanji, K., Bonilla, E., Pallotti, F., and Schon, E.A. 2002. Mitochondrial abnormalities in muscle and other aging cells: classification, causes, and effects. Muscle Nerve. 26:597-607.

9. Kim, K.M., and Shibata, D. 2002. Methylation reveals a niche: stem cell succession in human colon crypts. Oncogene. 21:5441-5449.

10. Schon, E.A and Manfredi, G. 2003. Neuronal degeneration and mitochondrial dysfunction. J. Clin. Invest. 111:303-312. doi:10.1172/ JCI200317741.

\title{
The TRAIL to arthritis
}

\author{
George C. Tsokos ${ }^{1}$ and Maria Tsokos ${ }^{2}$ \\ ${ }^{1}$ Department of Cellular Injury, Walter Reed Army Institute of Research, Silver Spring, \\ Maryland, USA \\ ${ }^{2}$ Laboratory of Pathology, National Cancer Institute, Bethesda, Maryland, USA
}

\begin{abstract}
Antigen-specific lymphocytes are involved in synovial proliferation within inflamed joints. Activated lymphocytes and synoviocytes from patients with rheumatoid arthritis express receptors that can bind TNF-related apoptosis-inducing ligand (TRAIL). A new study demonstrates that DCs pulsed with collagen and transduced with an adenovirus-based vector able to express TRAIL limit the incidence of arthritis in a model of collagen-induced arthritis and joint inflammation (see the related article beginning on page 1332). These results suggest that gene-modified cell therapy represents a therapeutic option for systemic rheumatic diseases.
\end{abstract}

J. Clin. Invest. 112:1315-1317 (2003). doi:10.1172/JCI200320297.

TNF-related apoptosis-inducing ligand (TRAIL) is a type II transmembrane protein that belongs to the TNF superfamily. It binds to death receptors (DRs) 4 and 5, two decoy receptors, and a soluble receptor called osteoprotegerin. The TRAIL signaling pathway was identified recently, and it has generated a great deal of interest since TRAIL induces apoptosis preferentially in tumor but not in normal cells, thus providing exciting opportunities for development of novel therapeutic strategies in cancer. TRAIL, like FasL, induces apoptosis by cross-linking and oligomerizing its receptors and forming a death-inducing signaling com-

Address correspondence to: George C. Tsokos, Walter Reed Army Institute of Research, Building 503, Room 1A32, Robert Grand Road, Silver Spring, Maryland 20910, USA. Phone: (301) 319-9911;

Fax: (301) 319-9133; E-mail: gtsokos@usa.net. Conflict of interest: The authors have declared that no conflict of interest exists. The opinions expressed herein are the private views of the authors and do not represent those of the Department of Defense.

Nonstandard abbreviations used: TNFrelated apoptosis-inducing ligand (TRAIL); death receptor (DR); doxycycline (DOX). plex through recruitment of an adapter molecule and the initiator caspase- 8 and subsequent mitochondria-dependent or -independent activation of the downstream effector caspase-3. Resistance of tumor cells to TRAIL has been associated either with low expression of its receptors or with defects in the downstream signaling (1).

Rheumatoid arthritis is a chronic inflammatory disorder that affects up to $1 \%$ of the population. The exact origin and pathogenesis of the disease are still unknown, and numerous diseasemodifying drugs and biologics have been tested. There is a significant need for increased efficacy and safety of these agents (2).

\section{TRAIL controls negative selection of $T$ cells in the thymus}

Recent reports have claimed a central role for TRAIL in thymocyte selection. TRAIL ${ }^{-/}$mice have larger thymi, and immature $\mathrm{CD}^{+} \mathrm{CD}^{+}$cells expressing high levels of heat-stable antigen are resistant to anti-CD3 antibody-mediated cell death. Similarly, TRAIL-/mice fail to reduce ovalbumin-specific cells following exposure to ovalbu- min. Both experiments clearly show that TRAIL is essential for negative selection of T cells in the thymus (3). In vitro, TRAIL blockade enhances the accumulation of concanavalin-stimulated spleen $\mathrm{T}$ cells into the S-G2/M cell cycle phase, supporting that TRAIL is important in the control of the lymphocyte cell cycle (4).

\section{TRAIL suppresses the development of arthritis}

TRAIL $^{-/-}$mice are sensitive to the development of collagen-induced arthritis, probably because they fail to delete relevant $T$ cell specificities and because they fail to properly silence activated $\mathrm{T}$ cells (3). As predicted, blockade of TRAIL with soluble DR5 administered systemically exacerbates arthritis, whereas direct transfer of a nonreplicative adenovirus expressing TRAIL into the joints of arthritic mice reduces arthritis (4). Injection of a TRAILexpressing adenovirus into IL- $1 \beta$ induced arthritic joints also significantly limits synovial proliferation (5).

An anti-TRAIL receptor antibody has been shown to be quite effective in treating bone-erosive disease in a model that involves transfer of fibrosarcoma cells into mice (6). However, antibodies to DRs, including those against CD95, may be associated with hepatotoxicity $(7,8)$, precluding their use in the treatment of tumors and autoimmune diseases.

\section{Collagen-pulsed TRAIL-expressing DCs suppress arthritis}

In this issue of the JCI, Liu et al. (9) report suppression of collagen-induced arthritis using DCs pulsed with collagen and transfected with an adenovirus-based vector expressing the TRAIL gene under the control of the doxycycline-inducible (DOX-inducible) tetracycline response element. The system offered two novel features: DCs were primed to recognize collagen-spe- 

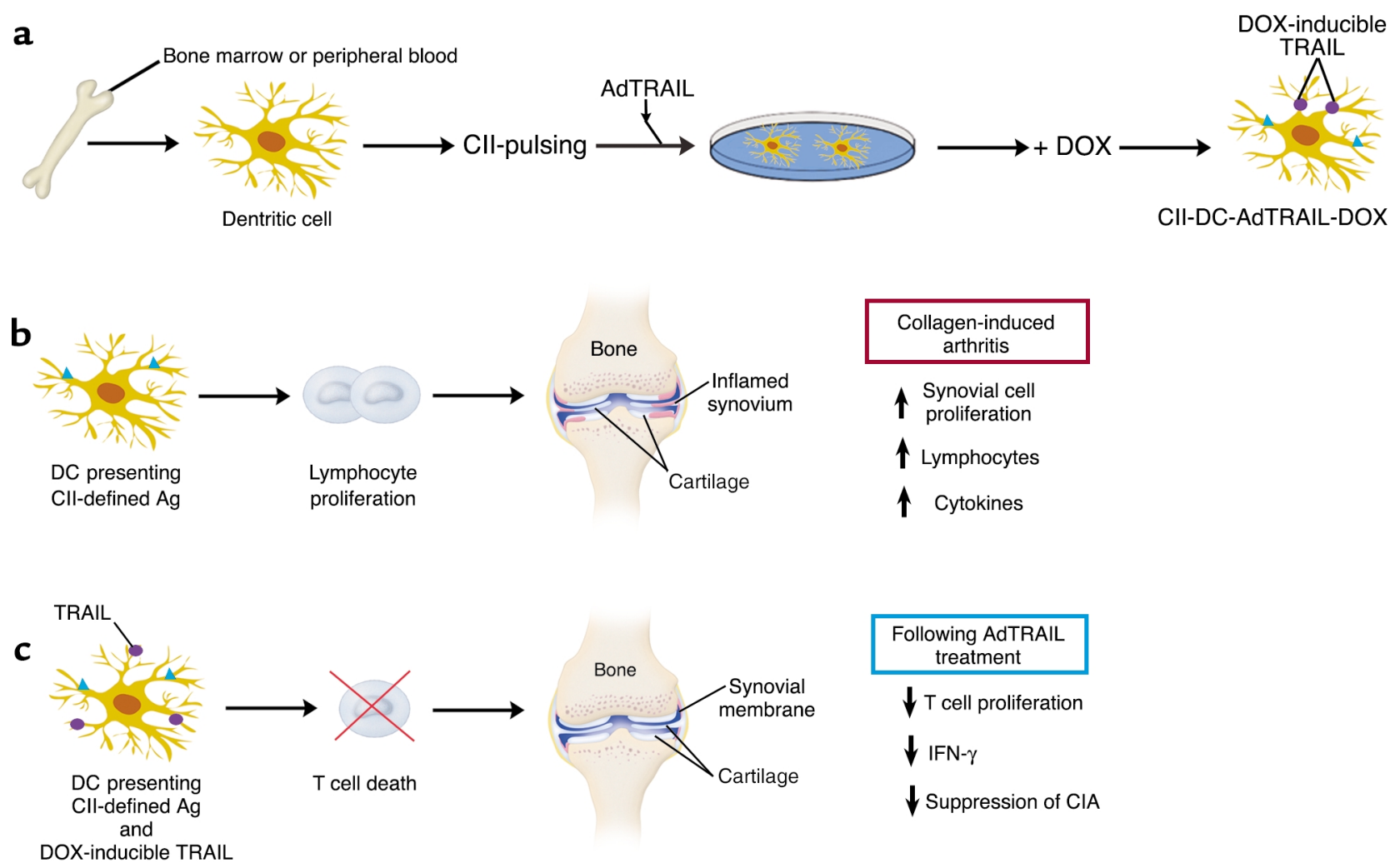

\section{Figure 1}

Gene-modified cell therapy in arthritis. (a) DCs prepared from either the peripheral blood or the bone marrow are pulsed with collagen type II (CII) and transfected with an adenovirus expressing TRAIL (AdTRAIL) to produce CII-DC-AdTRAIL-DOX. (b) Injection of CII into mice leads to a cascade of events that include lymphocyte activation, lymphokine production followed by synovial cell proliferation, and joint inflammation. (c) Infusion of DOX-inducible TRAIL-expressing DCs pulsed with CII suppresses collagen-induced arthritis (CIA) by eliminating CII-specific T cells.

cific $\mathrm{T}$ cells and to express TRAIL only when the expression vector was activated with DOX (Figure 1a). The extent of joint inflammation, measured as arthritic score, was limited significantly in the group of mice that received collagen-treated DCs expressing DOXcontrolled TRAIL (Figure 1, b and c). The authors also found that administration of non-collagen-primed TRAILexpressing DCs only delayed the appearance of arthritis and mildly reduced the arthritic score, a result that underlines the importance of antigen specificity. It was assumed that the response to other antigens was not affected; however, this was not tested. If antigen-pulsed TRAIL-expressing DCs also kill $\mathrm{T}$ cells specific for other antigens, then it is possible that ongoing immune responses, directed against, for example, the influenza virus, may be suppressed and silenced infections such as tuberculosis may flare. Anti-TNF treatment of patients with rheumatoid arthritis has been complicated by tuberculosis (2). This possible effect on ongoing immune responses can be projected from the fact that treatment with non-collagenpulsed TRAIL-expressing DCs still had some beneficial effect on the degree of arthritis development.

\section{Can TRAIL-expressing DCs be used in the treatment of rheumatoid arthritis?}

Synovial cells from patients with osteoarthritis express practically undetectable levels of DR5, whereas synovial cells from patients with rheumatoid arthritis express abundant levels of this receptor (6). Primary synovial cells from patients with rheumatoid arthritis succumb to TRAIL-mediated apoptosis if infected with a TRAILexpressing vector (5). The study by Liu et al. (9) does not address the localization of the infused modified DCs, although there is no doubt that these cells trafficked though the lymphoid organs and the inflamed tissues. Also, it is not known how long these DCs survived, and it is logical to assume that their half-life should be limited. If this novel delivery system is to be introduced for the treatment of rheumatoid arthritis, researchers will have to consider whether DCs should be additionally modified to limit their presence in the inflamed synovium. Synoviocytes may express molecules such as the newly described synoviolin, which helps with their identification (10). Finally, it is likely that more than one autoantigen is involved in human rheumatoid arthritis, and therefore the choice of the appropriate antigen(s) with which to pulse DCs may present additional challenges.

\section{Gene-modified DCs as therapeutic tools}

DCs represent the immunologist's dream cell, as they are able (a) to drive 
a Th1 or Th2 immune response (11), (b) to propagate an autoimmune response (12), and (c) to terminate the autoimmune response and re-establish tolerance (11). Genetically modified DCs have already been exploited therapeutically. DCs expressing FasL have been shown to increase survival of virally infected CNS cells by reducing antiviral $\mathrm{CD}^{+}$cells, suggesting that FasL-expressing DCs can control excessive tissue-damaging inflammation (13). Gene-modified DCs have also been used to control tumor growth (14). Recently, DCs have been used extensively in the treatment of autoimmune diseases with the hope of reversing established pathologic processes. DCs deficient in NF- $\mathrm{KB}$, following treatment with oligonucleotides, have been shown to prevent diabetes in NOD mice (15), and DCs infected with an IL-4-expressing adenovirus have been shown to traffic to the inflamed pancreas of NOD mice and suppress disease (16).

\section{Gene-modified T cell therapy in systemic autoimmune disease}

Correction of biochemical defects by means of gene transfer can be entertained to extend cell therapy to other autoimmune diseases. $T$ cells from patients with systemic lupus erythematosus have decreased levels of $\mathrm{T}$ cell receptor $\zeta$ chain, which has been replaced by the common $\gamma$ chain of the $\mathrm{Fc}$ receptors, which is responsible for hyperresponsiveness to CD3-mediated stimulation. Also, they express increased levels of the repressor cAMP response element modulator (CREM), which is responsible for the reduced production of IL-2 (17). Forced expression of $\zeta$ chain into $T$ cells restores normal responsiveness (18), and elimination of CREM by antisense technology has been shown to restore IL-2 production (19).
Gene therapy and gene-modified cell therapy are intensely studied in preclinical research and may soon be ready for clinical trials $(20,21)$. How should the results reported by Liu et al. (9) be translated to patients with rheumatoid arthritis? Will a trial using Ig-TRAIL be informative? Our discussion suggests that activated $T$ cells and synovial cells will be forced by TRAIL-expressing DCs to apoptose and that some clinical effect should therefore be observed. Will infusion of TRAIL-expressing DCs also eliminate cells that control latent infections? Should we use an IgTRAIL conjugate or TRAIL conjugated to an arthritogenic antigen(s)? Should nonviral, nonreplicative TRAIL-expressing vectors be injected into inflamed joints? Or should we infuse patients with DCs modified to express TRAIL? Despite recent advances in the treatment of rheumatoid arthritis, significant challenges remain that call for the development of more efficient treatments that lack side effects. The data presented by Liu et al. (9) offer new possibilities for cell-based treatment of patients with rheumatoid arthritis, provided we use the culprit autoantigens to provide specificity and solve problems inherent in gene transfer.

1. Mitsiades, N., Poulaki, V., Mitsiades, C., and Tsokos, M. 2001. Ewing's sarcoma family tumors are sensitive to tumor necrosis factor-related apoptosis-inducing ligand and express death receptor 4 and death receptor 5. Cancer Res. 61:2704-2712.

2. Smolen, J.S., and Steiner, G. 2003. Therapeutic strategies for rheumatoid arthritis. Nat. Rev. Drug Discov. 2:473-488.

3. Lamhamedi-Cherradi, S.E., Zheng, S.J., Maguschak, K.A., Peschon, J., and Chen, Y.H. 2003 Defective thymocyte apoptosis and accelerated autoimmune diseases in TRAIL-/- mice. Nat. Immunol. 4:255-260.

4. Song, K., et al. 2000. Tumor necrosis factor-related apoptosis-inducing ligand (TRAIL) is an inhibitor of autoimmune inflammation and cell cycle progression. J. Exp. Med. 191:1095-1104.

5. Yao, Q., et al. 2003. Intra-articular adenoviralmediated gene transfer of trail induces apopto- sis of arthritic rabbit synovium. Gene Ther. 10:1055-1060.

6. Ichikawa, K., et al. 2003. TRAIL-R2 (DR5) mediates apoptosis of synovial fibroblasts in rheumatoid arthritis. J. Immunol. 171:1061-1069.

7. Jodo, S., et al. 2003. Anti-CD95-induced lethality requires radioresistant Fcgamma RII+ cells. A novel mechanism for fulminant hepatic failure. J. Biol. Chem. 278:7553-7557.

8. Higuchi, H., Bronk, S.F., Taniai, M., Canbay, A., and Gores, G.J. 2002. Cholestasis increases tumor necrosis factor-related apoptotis-inducing ligand (TRAIL)-R2/DR5 expression and sensitizes the liver to TRAIL-mediated cytotoxicity. J. Pharmacol. Exp. Ther. 303:461-467.

9. Liu, Z., et al. 2003. CII-DC-AdTRAIL cell gene therapy inhibits infiltration of CII-reactive $\mathrm{T}$ cells and CII-induced arthritis. J. Clin. Invest. 112:1332-1341. doi:10.1172/JCI200319209.

10. Amano, T., et al. 2003. Synoviolin/Hrd1, an E3 ubiquitin ligase, as a novel pathogenic factor for arthropathy. Genes Dev. 17:2436-2449.

11. Steinman, R.M., Hawiger, D., and Nussenzweig, M.C. 2003. Tolerogenic dendritic cells. Annu. Rev. Immunol. 21:685-711.

12. Blanco, P., Palucka, A.K., Gill, M., Pascual, V., and Banchereau, J. 2001. Induction of dendritic cell differentiation by IFN-alpha in systemic lupus erythematosus. Science. 294:1540-1543.

13. Slifka, M.K., et al. 2003. Preferential escape of subdominant $\mathrm{CD} 8+\mathrm{T}$ cells during negative selection results in an altered antiviral $\mathrm{T}$ cell hierarchy. J. Immunol. 170:1231-1239.

14. Okada, N., et al. 2003. Dendritic cells transduced with gp100 gene by RGD fiber-mutant adenovirus vectors are highly efficacious in generating anti-B16BL6 melanoma immunity in mice. Gene Ther. 10:1891-1902.

15. Ma, L., et al. 2003. Prevention of diabetes in NOD mice by administration of dendritic cells deficient in nuclear transcription factor-kappaB activity. Diabetes. 52:1976-1985

16. Feili-Hariri, M., et al. 2003. Dendritic cells transduced to express interleukin-4 prevent diabetes in nonobese diabetic mice with advanced insulitis. Hum. Gene Ther. 14:13-23.

17. Tsokos, G.C., Nambiar, M.P., Tenbrock, K., and Juang, Y.T. 2003. Rewiring the T-cell: signaling defects and novel prospects for the treatment of SLE. Trends Immunol. 24:259-263.

18. Nambiar, M.P., et al. 2003. Reconstitution of deficient $T$ cell receptor zeta chain restores $T$ cell signaling and augments $\mathrm{T}$ cell receptor/CD3 induced interleukin-2 production in patients with systemic lupus erythematosus. Arthritis Rheum. 48:1948-1955.

19. Tenbrock, K., Juang, Y.-T., Gourley, M.F., Nambiar, M.P., and Tsokos, G.C. 2002. Antisense cyclic adenosine 5 -monophosphate response element modulator up-regulates IL-2 in T cells from patients with systemic lupus erythematosus. J. Immunol. 169:4147-4152.

20. Tsokos, G.C., and Nepom, G.T. 2000. Gene therapy in the treatment of autoimmune diseases. J. Clin. Invest. 106:181-183.

21. Robbins, P.D., Evans, C.H., and Chernajovsky, Y. 2003. Gene therapy for arthritis. Gene Ther. 10:902-911. 